\title{
Molecular interrelations in the Melodinus alkaloids
}

\author{
László F. Szabó \\ Department of Organic Chemistry, Semmelweis University, Högyes utca 7, \\ H-1092 Budapest, Hungary \\ E-mail: Szalasz@szerves.sote.hu
}

Dedicated to Prof. Atta-Ur-Rahman on the occasion of his $60^{\text {th }}$ birthday

\begin{abstract}
Structural and chemotaxonomical data as well as consideration of standard organic reaction mechanisms provided a coherent picture about the probable formation of the small but characteristic group of Melodinus alkaloids in the plant family Apocynaceae. All 14 alkaloids could be classified into the melodan skeleton and its rearranged version and derived from 18,19didehydrotabersonine as parent compound.
\end{abstract}

Keywords: Indole alkaloids, reaction mechanism, chemotaxonomic, database, computersearched

\section{Introduction}

The plant cells may be considered as chemical reactors in which the alkaloids as products are formed from precursors as educts. In the past more than sixty years a large number of indole alkaloids were isolated, and their three-dimensional structures, chiral and chemical properties established with high accuracy. Several points of their biogenesis were likewise detected, ${ }^{1,2,3}$ and approaches proposed for construction a biogenetic-type system of them. ${ }^{1,4,5}$ However, the methods applied for their biogenesis are rather complicated and time-consuming, the labelling technique is not always reliable, ${ }^{6}$ the enzymatic and genetic approaches do not give detailed informations about the molecular mechanisms, and even the biomimetic-type synthetic methods imitate Nature only roughly. Therefore the experimental data were not sufficient to detect the general principles and detailed molecular interrelations among the individual natural products.

Fortunately, in the last decade, the great databases and their computer-assisted searching presented a further tool to investigate the formation of the natural products, which seemed to be applicable in the class of indole alkaloids based on secologanin. More than 2500 individual alkaloids are formed from secologanin $\mathbf{1}$ and tryptamine $\mathbf{2}$ mainly in three plant families 
Rubiaceae (RUB), Loganiaceae (LOG) and Apocynaceae (APO). It became clear, that the isolation of such a huge number of compounds from a strictly limited group of related plant families, the common availability of homo- and heterosesqui- and dimer alkaloids, the presence or absence of structural elements, ring systems and functional groups, even of a double bond or hydroxy group in the same family, genus or species do represent a strong coherent power. The computer-assisted search and work-up of the structural and chemotaxonomical data should facilitate to sketch a consistent picture of the most important molecular connections among the individual alkaloids. The way is marked by stable classical compounds having well-established structures, which were actually isolated from natural sources. With carefull application of the basic organic reaction mechanisms, these guide-posts may help to postulate the selection of labile and reactive key-intermediates, which are hypothetic or exist in minute amount in the plants. These structures then mutually confirm or exclude each other.

In the frame of our work on the field of the chemistry of secologanin, ${ }^{7}$ several problems were investigated. The configuration of $\mathrm{C}-3$ and the total stereochemistry of strictosidine was rigorously proved. ${ }^{8}$ It was demonstrated why are homochiral all type I alkaloids at $\mathrm{C}-15$ and most of them even at C-3. ${ }^{9}$ Formation of the possible aglucones after deglucosylation of strictosidine was analyzed by graph analysis. ${ }^{10}$ A general scheme was proposed for the formation of the type I indole alkaloids which represent the largest subclass of indole alkaloids and are produced immediately from the common precursor strictosidine 3. ${ }^{11}$ Meanwhile the question arose whether would it be possible to analyze this problem in a small, more specific group, near at the end of the molecular evolution, in which, however, the structural changes are deeper and more complicated. The group of Melodinus alkaloids seemed to be appropriate for such a study.

In the present work such compounds are considered as indole alkaloids, which can be derived from 1 and 2 irrespectively of the presence or absence of an indole ring (Scheme 1). However, the compounds having not one secologanin subunit and one tryptamine subunit as precursors (i. e. the sesqui- and dimer alkaloids) were temporarily disregarded.

The necessary structural, chemical and chemotaxonomical data were taken from the database Dictionary of Natural Products Version 15.1 (in the following DNP), ${ }^{12}$ completed, if necessary, with data of the Beilstein Crossfire and the Chemical Abstracts on line databases. The number of compounds in Scheme 2 and elsewhere in the text represent isolated compounds in the DNP database. In the structural formulae and throughout the text, the biogenetic numbering system shown in structure of strictosidine $\mathbf{3}$ was used. ${ }^{13}$ The only exceptions are the building blocks $\mathbf{1}$ and $\mathbf{2}$ which were numbered as indicated in their own formulas.

The DNP database contains 14 alkaloids of this type (type of compound code in DNP: VX5580), and in this paper the melodan alkaloid name will be applied for them. They were isolated from the Apocynaceae plant family i. e. from one of the main sources of indole alkaloids, however, they have a quinoline rather than an indole ring system. In addition to chemotaxonomical arguments, their structure can easily be derived from tryptamine (and secologanin, of course) by extension of the pyrrol into a pyridine ring (see later). The cinchonan and camptothecan alkaloids (24 and 36 natural products, respectively) are well-known examples 
of such "quinolino-indole alkaloids" and 16 similar compounds were sporadically isolated in other subclasses as well. It means that presently about 90 "quinolino-indole"alkaloids are known.

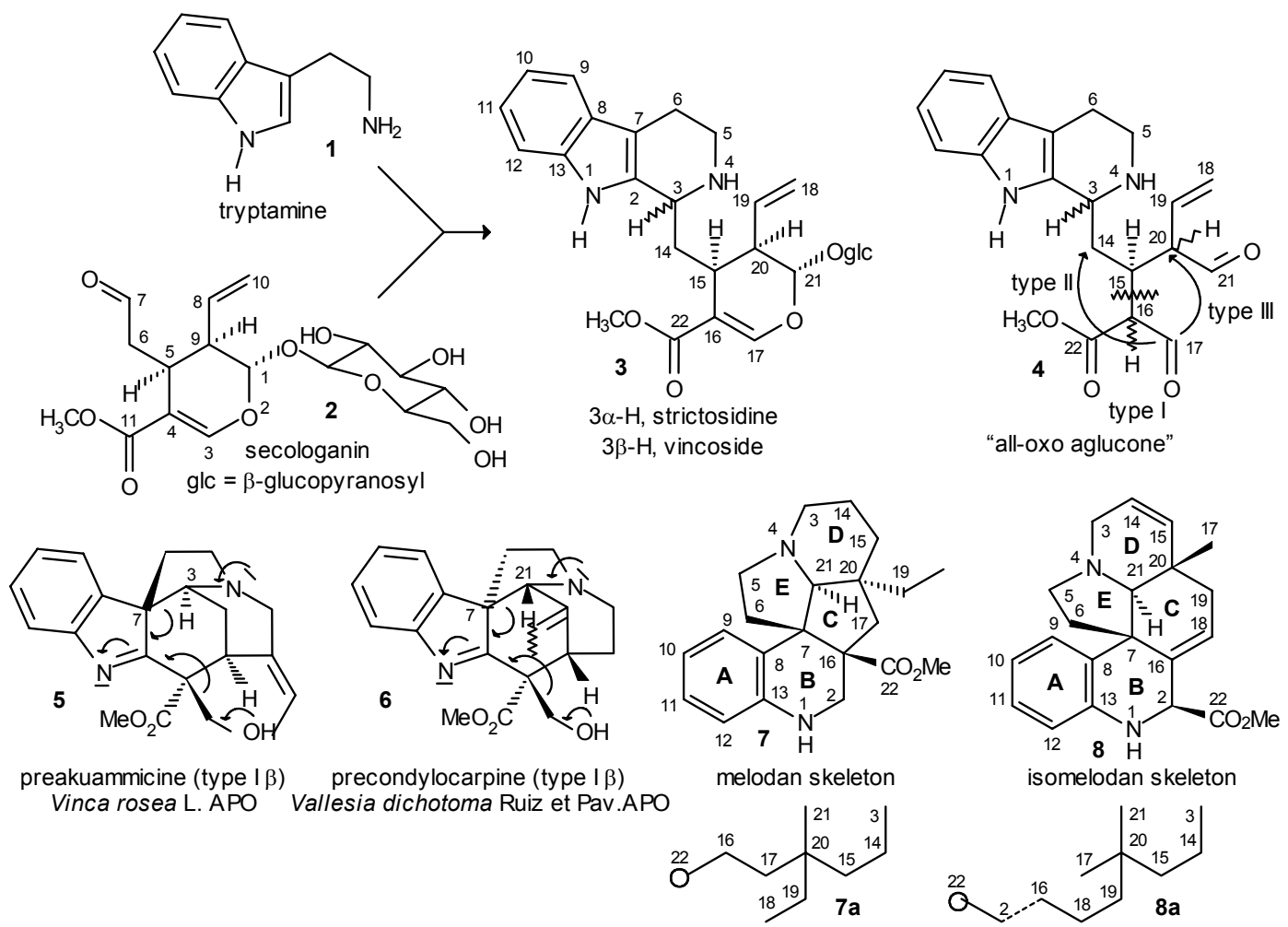

Scheme 1. The coupling reaction and some key structures.

\section{Genealogy of the Melodan Alkaloids}

The first-type melodan alkaloids (except meloscandonine and its epimer 19, see later) (Scheme 3) have a vinyl group (C-18-C-19), which comes evidently from secologanin (C-8-C-10), and has an important role in the biogenesis, but, during the long biosynthetic pass, it is generally transformed into other structural elements (e. g. 1- or 2-hydroxyethyl, acetyl group, etc.). ${ }^{15-21}$ Therefore, except the first-type melodan alkaloids, no alkaloid of the plumeran subclass contains the vinyl group in intact form. However, the presence of the 18,19-unsaturation in the previous phases of the biogenesis is strongly supported by the functional groups mentioned above and by further oxygen-containing cycles, which were developed evidently from the vinyl group. 


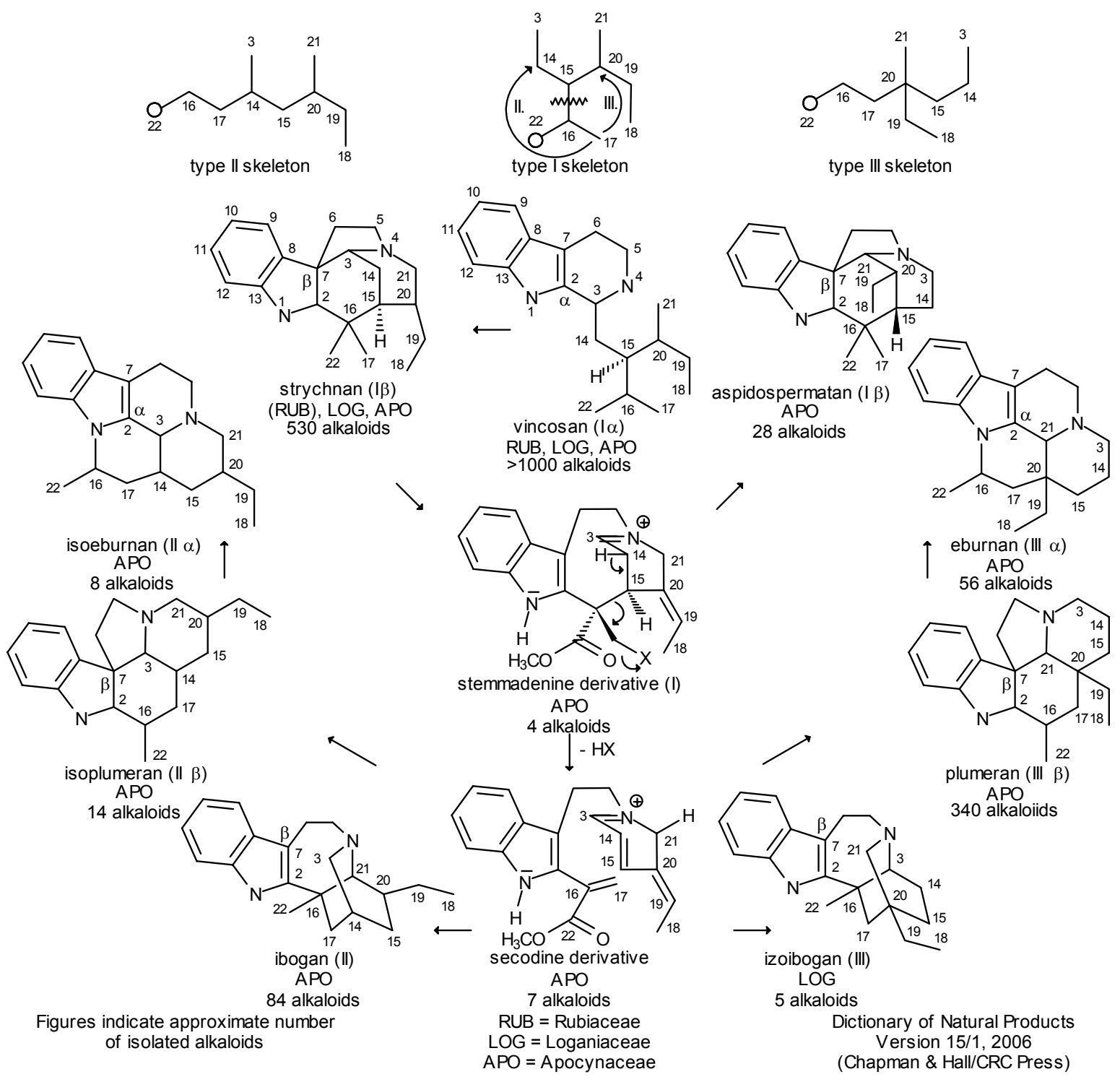

Scheme 2. Indole alkaloids derived from secologanin and having an intact tryptamine aubunit

For our special purpose, a new bioorganic-type system of the structures was constructed, ${ }^{9}$ and its simpified version is shown in Scheme 2. The great majority of the indole alkaloids can be classified into one of the three main carbon skeletons. Type I skeleton has the carbon framework of secologanin in intact, the other two in rearranged form. As shown in the all-oxo-aglucone 4 (in Scheme 1) and in Scheme 2, these latter skeletons can be derived by dissociation of the C15-C-16 bond, and followed by subsequent association of C-17 to C-14 in the type II, and to C20 in the type III skeleton. An experimental analog of this bond cleavage was presented earlier. ${ }^{14}$ The formation of the melodan alkaloids is the result of a long molecular evolution, as is briefly outlined in Scheme 2. In the coupling reaction of $\mathbf{1}$ and $\mathbf{2}$, strictosidine $\mathbf{3}$ is formed, which, after deglucosylation, affords the large subclass of the type I $\alpha$ (vincosan) alkaloids, i. e. compounds 
having C-3 of the secologanin subunit attached to the $\alpha$ position of the indole ring. In the next phase of the evolution, $\mathrm{C}-3$ is shifted to the $\beta$ position of the indole ring, and with simultaneous association of $\mathrm{C}-16$ to $\mathrm{C}-2$, the first group of the type $\mathrm{I} \beta$ alkaloids (strychnan alkaloids) is formed. In preakuammicine (5 in Scheme 1), i. e. in the first representative of strychnan alkaloids, the cleavage of the bond C-3-C-7 may give rise a stemmadenine derivative, which can further be transformed by isomerization and recyclization into precondylocarpine ( 6 in Scheme $1)$, the first representative of the second group of type I $\beta$ alkaloids (aspidospermatan alkaloids). Alternatively, a further cleavage of bond C-15-C-16 of the stemmadenine derivative gives secodine derivatives which are key structures in the formation of the type II and III alkaloids. The mechanism of these latter transformations is not clear yet, nevertheless it can most easily be interpreted by intramolecular Diels-Alder reactions between the conjugated double-bond systems present in hypothetic secodine intermediates. In both (type II and type III) classes two types of pentacyclic ringsystems, i. e. bridged (ibogan and isoibogan) and fused (plumeran and isoplumeran) alkaloids are formed. Most indole alkaloids having a rearranged secologanin subunit can be classified into one of these ringsystems. In the fused systems the evolution runs one phase further: the ligand of C-7 (C-3 or C-21) can return into the $\alpha$ position of the indole ring with simultaneous cleavage of bond $\mathrm{C}-2-\mathrm{C}-16$, and the II $\alpha$ (isoeburnan) and III $\alpha$ (eburnan) alkaloids, respectively, are formed. As it will be shown, all melodan alkaloids can be derived chemically from 18,19-didehydrotabersonine 9, i. e. a III $\beta$ type (plumeran) structure, which should be considered also as the parent compound of all plumeran type alkaloids.

The small group of melodan alkaloids may be divided into two subgroups. 10 members of the first-type melodan alkaloids having the melodan skeleton 7 (in Scheme 1) were isolated from a single species Melodinus scandens Forst. (and in addition, scandine and derivatives from other Melodinus species as well), and contain the type III secologanin subunit in unchanged form and fused arrangement to the tryptamine subunit (plumeran alkaloids). Neither their indole nitrogen nor their benzene ring has any standard substituent. It should be noted that the Melodinus species contain many other plumeran alkaloids as well, e. g. tabersonine derivatives.

The 4 members of the second-type melodan alkaloids (in Scheme 1) were isolated not from Melodinus species but from a not-specified "malayan Kopsia species" by the research group of T.-S. Kam. However, unlike to the other Kopsia alkaloids, these compounds have a further rearranged variant of the melodan skeleton (isomelodan 8 in Scheme 1). For their formation an intact vinyl group is crucial. In addition, their indole nitrogen has a methoxycarbonyl and their benzene ring in 10,11-position a methylenedioxy group as standard substituents. 


\section{Derivation of the First-type Melodan alkaloids}

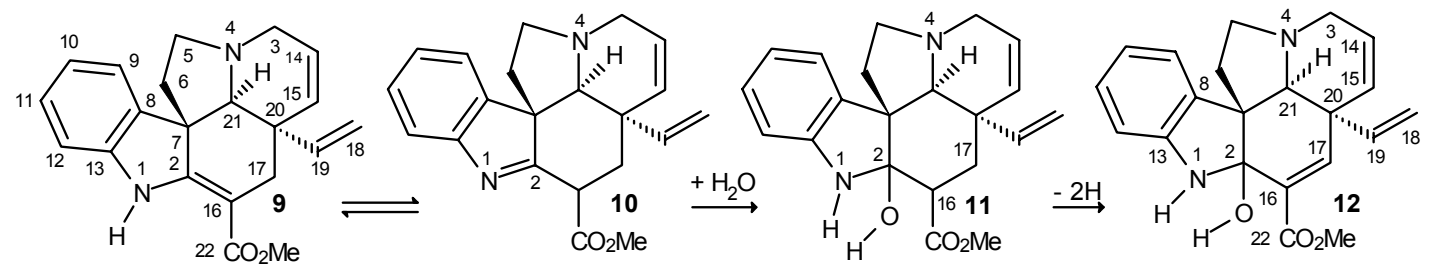

18,19-didehydrotabersonine

[tabersonine, GQX60

and its 18 natural derivatives

from Amsonia, Melodinus

(2)
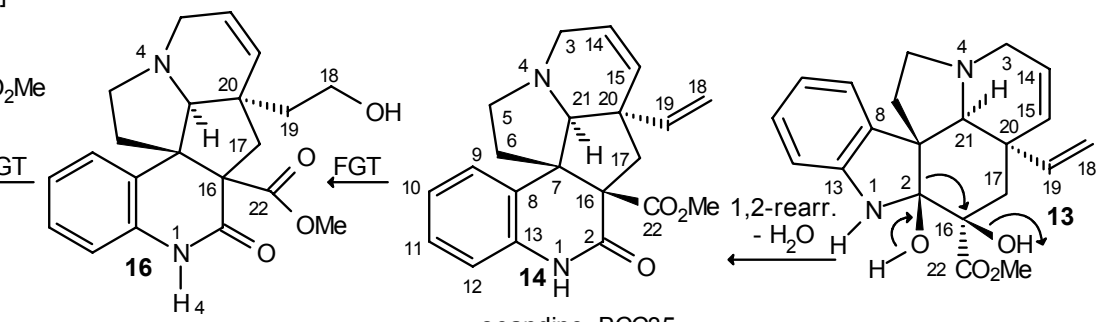

ester

FGT = functional group transformation Melodinus scandens Forst

$\sim \mathrm{N}-4$ oxide, MPV77 $\quad+\mathrm{H}_{2} \mathrm{O}$

Melodinus fusiformis Champ. ex Benth. $-\mathrm{MeOH}$

$\sim$ 14,15-epoxide, LXG40

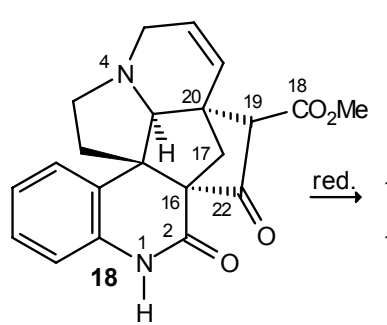

Melodinus hemsleyanus Diels

10-hydroxy, LCL12

1. epoxid
2. red.

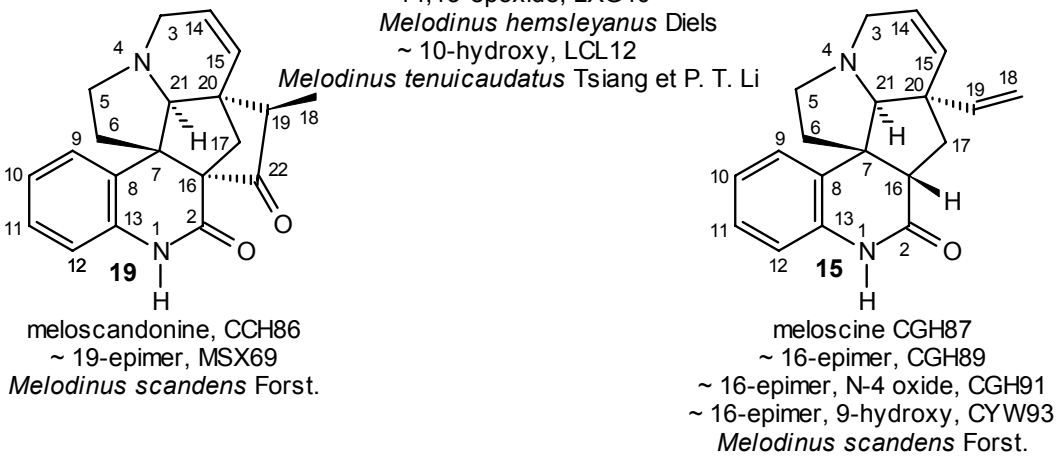

Scheme 3. Formation of Melodan alkaloids. Part 1.

The chemical structures and the chemotaxonomical conditions suggest that scandine $\mathbf{1 4}$ is the key compound in the biosynthesis of these alkaloids, because it contains all carbon atoms of the secologanin subunit and no additional one. Therefore, the first task is to interprete the formation of the quinoline ring system in $\mathbf{1 4}$ from the original indole subunit in $\mathbf{9}$. Extension of the pyrrol ring into a pyridine ring requires the shift of $\mathrm{C}-7$ from $\mathrm{C}-2$ to $\mathrm{C}-16$ (1,2-rearrangement) with simultaneous formation of a carbonyl function at C-2. In the hydrated form $\mathbf{1 1}$ of the tautomer $\mathbf{1 0}$ of 18,19-didehydrotabersonine 9, partial structural conditions for a special type of pinacol rearrangement are already present. However, an appropriate electrophilic centre at C-16 still fails. Presence of a double bond into position C-16-C-17 of $\mathbf{1 2}$ or even a hydroxyl group at C-16 (and $\mathrm{C}-17$ ) in $\mathbf{1 3}$ by (ep)oxidation (and subsequent partial reduction) can provide this element. The double bond in 16,17-position is present in kopsidasine, kopsijasmine and their derivatives 
(8 natural products), and a hydroxy group at C-16 in kopsaporin, kopsinaline and singapurensine derivatives (20 natural products), so this presupposition is a reasonable requirement. Finally, the pinacol rearrangement, indicated by curved arrows in $\mathbf{1 3}$ and probably catalyzed by proton affords scandine 14. This rearrangement is common at different levels of the biogenesis of the indole alkaloids, e. g. in the formation of the large group of oxindole and indoxyl alkaloids (116 and 17 natural products, respectively), ${ }^{22-24}$ and sporadically in other subclasses (10 natural products) as well. Of course, the exact form and order of the proposed transformations can not be prognosed.

The next simple step is the removal of the methoxycarbonyl group (containing C-22) of 14 by hydrolytic decarboxylation. This step is likewise common in the biogenesis of the indole alkaloids, both in the early and the late phases of the molecular evolution. The process is certainly catalyzed by enzymes and in the present case affords meloscine $\mathbf{1 5}$ (and its derivatives).

The isolation of meloscandonine $\mathbf{1 9}$ from the same species indicates the operation of a more complicated process. In the presence of a methoxycarbonyl group, formation of a new fivemembered carbacycle with a keto group suggests the role of an ester condensation, for which, however, it is necessary to activate $\mathrm{C}-19$ by construction of a second strong electron-attracting group from the vinyl unit of 14. The proposed hydroxyethyl and methoxycarbonylmethyl structural elements as temporary functional groups in $\mathbf{1 6}$ and 17, respectively are known in the limaspermidine and the cylindrocarine derivatives ( 8 and 17 natural compounds, respectively). The ester condensation shown in $\mathbf{1 7}$ is one of the main reactions in the formation of new carboncarbon bonds for construction of longer carbon chains and larger cycles. ${ }^{25}$ Reduction of the temporarily formed methoxycarbonyl group in $\mathbf{1 8}$ would afford meloscandonine $\mathbf{1 9}$ and its C-19 epimer. Change of configuration at C-16 from 14 to 19 is not clear.

\section{Derivation of the Second-type Melodan alkaloids}

Interpretation of the formation of second-type melodan alkaloids (Scheme 4) is a more difficult problem. $^{26,27}$

Methoxycarbonyl group is attached to the indole nitrogen in 91 indole alkaloids, nearly all of them are plumeran alkaloids (type III $\beta$ skeleton), and 84 were isolated from Kopsia species (most of them by T.-S. Kam's group!). Consequently, introduction of the methoxycarbonyl group to N-1 takes place probably in a late phase of the molecular evolution. In addition, the methylenedioxy group at position 10,11 is likewise characteristic for the Kopsia species (29 alkaloids). However, its tight connection to the benzene ring suggests its entrance already in the formation of the simple tryptamine derivatives.

The difference between the melodan 7 and isomelodan $\mathbf{8}$ skeleton (Scheme 1) is special: the five-membered cycle $\mathbf{C}$ of the first-type alkaloids is extended into a six-membered one in the second-type alkaloids according to the fact, that the positions of the intracyclic $\mathrm{C}-17$ and the exocyclic C-18-C-19 subunits are exchanged. The differences are clearly shown also in the original (7a) and the rearranged (8a) type III secologanin subunits (Scheme 1). A further 
problem is posed by transfer of the original methoxycarbonyl group and construction of an additional one.
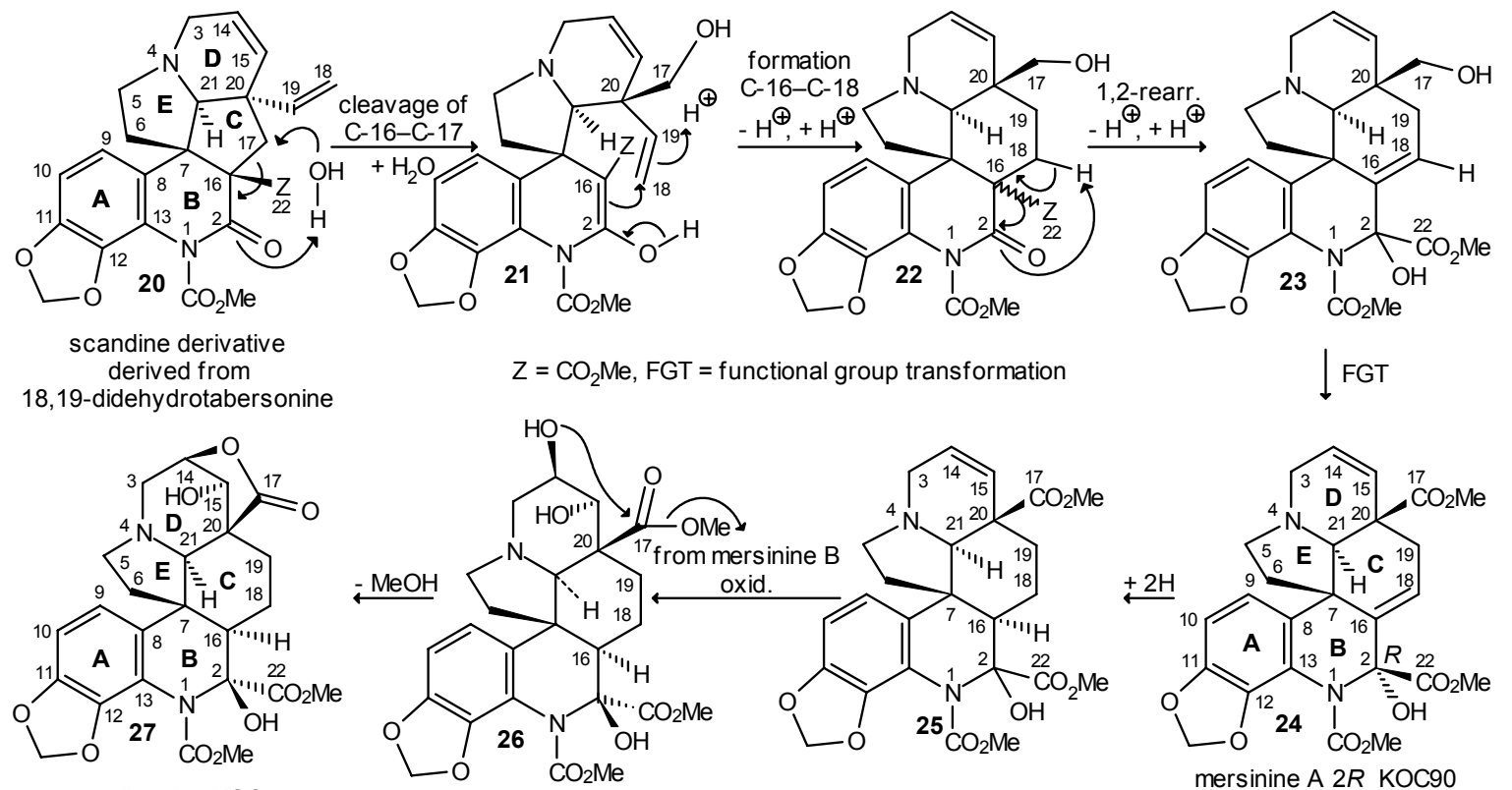

mersiloscine KOC89 malayan Kopsia sp.
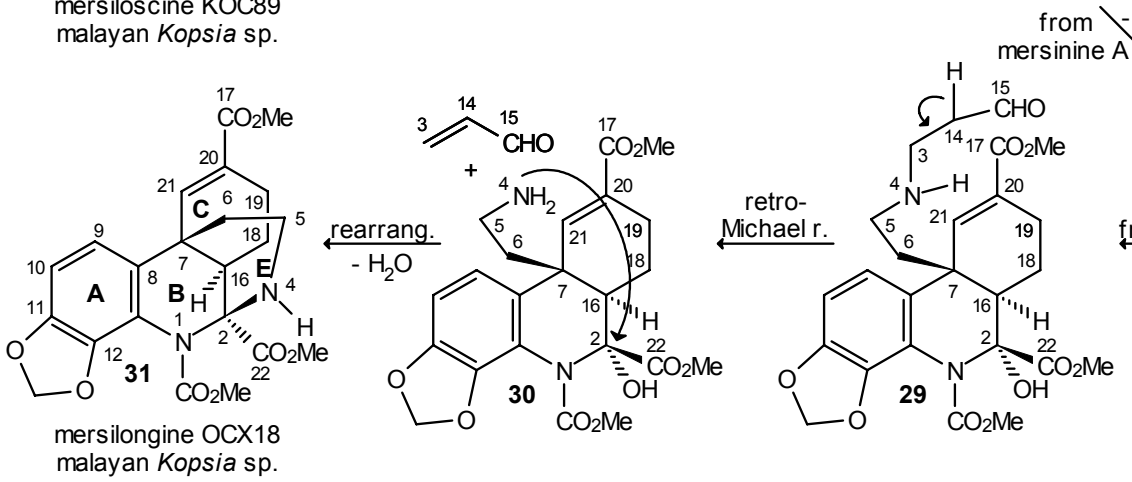

rsinine A $2 R$ KOC90 malayan Kopsia sp.

\section{Scheme 4. Formation of Melodan alkaloids. Part 2.}

The following proposition was developed for interpretation of these changes. The precursor should be a scandine derivative $\mathbf{2 0}$ having the standard functional groups and the vinyl group precursor. Undoubtedly, the crucial problem is the rearrangement of the type III skeleton. In the first step the bond $\mathrm{C}-16-\mathrm{C}-17$, which is already weakened by two strong electron-attracting groups, should be cleaved hydrolytically according to the curved arrows in $\mathbf{2 0}$. Then, by a minimum rotation around $\mathrm{C}-20-\mathrm{C}-21$, the steric position of the two ligands $\mathrm{C}-17$ and $\mathrm{C}-18-\mathrm{C}-19$ can be exchanged, and in the second step, suggested likewise by curved arrows in 21, the new bond C-16-C-18 may be formed in $\mathbf{2 2}$ in a special type of Michael addition. Though the double bond taking part in this reaction, is not activated, the minimum rotational motion and the driving force of these two steps coming from the transformation of the strained five-membered ring $\mathbf{C}$ in 
20 into a less strained six-membered one in $\mathbf{2 2}$ could facilitate it. Finally, in the third step, the methoxycarbonyl group should be moved in a 1,2-rearrangement from C-16 in 22 to C-2 in 23 with the assistance of the formation of a double bond, as indicated again by curved arrows. This rearrangement is a remote analogue of the pinacol rearrangement, and hereby C-22 which was part of the secologanin subunit up till now, is now attached to the tryptamine subunit.

In absence of any extra carbon atom in $\mathbf{2 0}$ and 21, appearance a new methoxycarbonyl group in 24 might be interpreted only by the refashion of the hydroxymethyl group containing C-17. It can not be prognosed, on which level would it take place in the reaction sequence, however the availability of compounds having hydroxymethyl and methoxycarbonyl groups in related species (Aspidosperma cylindrocarpon Müll. Arg. and A. limae Woodson in Apocynaceae) supports the reality of this transformation. The sequence of these reactions would afford mersinine $\mathrm{A}$ and its 19-epimer mersinine B 24.

The further transformations to mersiloscine $\mathbf{2 7}$ and mersilongine $\mathbf{3 1}$ are already less problematic. As in both final products the C-16-C-18 double bond is absent, it should be saturated somewhere in the sequence by a usual biogenetic hydrogenation. In addition, the formation of mersiloscine $\mathbf{2 7}$ requires the construction of a hydroxylactone subunit. The necessary elements are already present in mersinine 24. The C-14-C-15 double bond in 25 could easily be transformed into a geminal diol system $\mathbf{2 6}$ by direct oxidation or through the epoxide. Both proposed functional groups are common in the plumeran alkaloids. Spontaneous lactonization would afford immediately mersiloscine $\mathbf{2 7}$ as a final product.

Mersilongine 31 has a special structure, the formation of which needs further elaboration. ${ }^{27}$ The original cycle $\mathbf{D}$ in $\mathbf{2 4}$ should be destroyed by removal of its three atoms (C-3, C-14 and C15) and cycle $\mathbf{E}$ transformed by shifting of N-4 from C-21 to C-2. The key step of the reaction sequence is the introduction of an appropriate functional group. A hydroxy group at C-15 seems to be useful in this case. In 25, regioselective hydration of the double bond or even regioselective hydrogenolysis of the supposed epoxide intermediate might be appropriate methods which are well known possibilities in the plumeran alkaloids. As shown by curved arrows in 28, the fragmentation involving $\mathrm{N}-4$ and the hydroxy group of C-15 results in the cleavage of both bonds N-4-C-21 and C-15-C-20 which is necessary for the subquent transposition of N-4. Simultaneously, in 29 the structural conditions of a retro-Michael reaction are built up. After removal of the $\mathrm{C}_{3}$ unit in this latter reaction, $\mathbf{3 0}$ can be formed, in which N-4 would easily be shifted to C-2 formally by elimination of water, and $\mathbf{3 1}$ afforded. In this last step of derivation the original five-membered ring $\mathbf{E}$ in $\mathbf{2 8}$ is transformed into a six-membered one in $\mathbf{3 1}$, and hereby the last element of steric strain has been removed from the polycyclic structure. If we consider the configuration of C-2 in $\mathbf{2 7}$ and 31, and suppose inversion of the configuration of C-2 in recyclization $\mathbf{3 0} \rightarrow \mathbf{3 1}$, it might be concluded that $\mathbf{2 7}$ should be formed from mersinine $\mathrm{B}$ and 31 from mersinine A. 


\section{Conclusions}

Our initial expectation to establish molecular interrelations among individual indole alkaloids by combination of computer-assisted search of chemotaxonomical properties and standard organic reaction mechanisms, seems to be successful. It was possible to obtain a coherent picture about the possible formation of the small but strongly diverse group melodan alkaloids. Of course, it could not be expected to propose either detailed reaction conditions (reagent, catalyst, solvent, etc.) or sometimes even the exact order of the steps. However, most of the proposed steps and supposed intermediates or partial structures find their analogues in other subclasses and subgroups of the collection of indole alkaloids, which increases the consistency of the system.

\section{Acknowledgements}

The intellectual and finantial support of the Central Library of the Semmelweis University and its director in-chief, Dr. Livia Vasas is thankfully acknowledged.

\section{References}

1. Atta-Ur-Rahman, Anwer B. Biosynthesis of Indole Alkaloids, Clarendon Press: Oxford, 1983.

2. Cordell, G. A. Lloydia 1974, 37, 219.

3. Beke, Gy.; Patthy-Lukáts, Á.; Podányi, B; Szabó, L. F. Chirality, 2001, 13, 483.

4. Kisakürek, M. V.; Leeuwenberg, A. J.; Hesse, M. Alkaloids: Chemistry and Biological Properties, Vol. 1, Pelletier, S. W., Ed., John Wiley and Sons: New York, 1983, pp 211-376.

5. Hesse, M. Alkaloids, Verlag Helvetica Chimica Acta: Zürich, 2002.

6. Contin, A.; van der Heijden, R.; Lefeber, A. W. M.; Verpoorte, R. FEBS Lett. 1998, 434, 413.

7. Szabó, L. F. Studies in Natural Products Chemistry, Vol. 26. Bioactive Natural Products. Part G. Atta-Ur-Rahman, Ed., Elsevier: Amsterdam, 2002; pp 95-148.

8. Patthy-Lukáts, Á.; Károlyházy, L.; Szabó, L. F.; Podányi, B. J. Nat. Prod. 1997, 60, 69.

9. Beke G.; Károlyházy L.; Patthy-Lukáts Á.; Podányi B.; Szabó L. F. Progress in Biological Chirality, Pályi, Gy.; Zucchi, C.; Caglioti, L. Eds., Elsevier: Amsterdam, 2004; pp 377-395.

10. Károlyházy, L.; Patthy-Lukáts, Á.; Szabó, L. F. J. Phys. Org. Chem. 1998, 11, 622.

11. Szabó, L. F. J. Phys. Org. Chem. Accepted for publication.

12. Dictionary of Natural Products, Version 15.1, Chapman and Hall/CRC: Boca Raton, 2006.

13. Le Men, J.; Taylor, W. I. Experientia 1965, 21, 508.

14. Károlyházy, L.; Patthy-Lukáts, Á.; Szabó, L. F.; Podányi, B. Tetrahedron Lett. 2000, 41, 1575 . 
15. Plat, M.; Hachem-Mehri, M.; Koch, M.; Scheidegger, U.; Potier, P. Tetrahedron Lett. 1970, 11,3395 .

16. Mehri, H.; Plat, M.; Potier, P. Annales Pharmaceutiques Francaises, 1971, 29, 291.

17. Guo, L.; Zhou, Y. Phytochemistry, 1993, 34, 563.

18. Bernauer, K.; Englert, G.; Vetter, W.; Weiss, E. Helv. Chim. Acta, 1969, 52, 1886.

19. Mehri, H.; Diallo, A. O.; Plat, M. Phytochemistry; 1995, 40, 1005.

20. Zhou, Y. L.; Ye, J. H.; Li, Z. M.; Huang, Z. H. Planta Med. 1988, 54, 315.

21. He, X.; Zhou, Y.; Huang, Z.; Chem. Abstr. 116, 191092c.

22. Shellard, E. J.; Sarpong, K. Planta Med. 1971, 20, 167.

23. Shellard, E. J.; Houghton, P. J. Planta Med. 1972, $21,16$.

24. Ref. 1, p. 71-74.

25. Dewick, P. M. Medicinal Natural Products. A Biosynthetic Approach. John Wiley and Sons: New York, 1997; pp 13-16.

26. Kam, T. S.; Subramaniam, G.; Lim, T. M. Tetrahedron Lett. 2001, 42, 5977.

27. Kam, T. S.; Subramaniam, G. Tetrahedron Lett. 2004, 45, 3521. 